\title{
Astrometric Measurements of Rarely-Observed Minor Planets
}

Roger Chanal

Nurol, F-43110 Aurec sur Loire, France

\begin{abstract}
The 1986 Minor Planet Ephemerides call observers' attention to the need for astrometric observations of 120 objects on the "critical list". Their magnitudes are generally between 15 and 18 at opposition. The author's programme of observation with simple means was described. The equipment used was a Newtonian $41-\mathrm{cm}$, f/4.8 reflector, a standard camera back, and Kodak TP 2415 film, hypersensitized in forming gas. Out of 25 observations attempted, 20 photographs were obtained, 8 of which were of sufficient quality for the positions to be measured and to be published in Minor Planet Circulars. On two photographs, the minor planet (1982 KM) was detected, but there were no suitable reference stars within the field. (The lack of reference stars brighter than mag. 10 in certain regions should be noted.) The return of measured positions versus photographs obtained (40\%) appears very acceptable.
\end{abstract}

\section{Video Recordings of Asteroidal Occultations}

Roland Boninsegna ${ }^{1}$ and Jean Lecacheux ${ }^{2}$

1 rue de Mariembourg, 33, B-6381 Dourbes, Belgium

2 Observatoire de Paris, F-92195 Meudon Principal Cedex, France

\section{Abstract}

Text not received

Video recordings of two occultations were shown, the first concerning the star AGK3+29 0398 and (93) Minerva on 1982 November 22, 03:31 UT, using the $1-\mathrm{m}$ telescope at Pic du Midi. The occultation was also observed in Spain and in the U.S.A. The second event involved AGK3+17 1309 and (146) Lucina on 1982 April $18,20: 23 \mathrm{UT}$, using the 1-m telescope at Meudon. A short secondary occultation $(0.6 \mathrm{sec})$ was observed, not caused by the minor planet. A possible interpretation is a small satellite $(5.7 \mathrm{~km}$ minimum diameter $)$ near Lucina.

\section{References}

Arlot, J.-E., et al.: Icarus, 61, 224, 1985

Millis, R.-L., et al.: Icarus, 61, 124, 1985

Arlot, J.-E., et al.: Icarus, 61, 224, 1985 